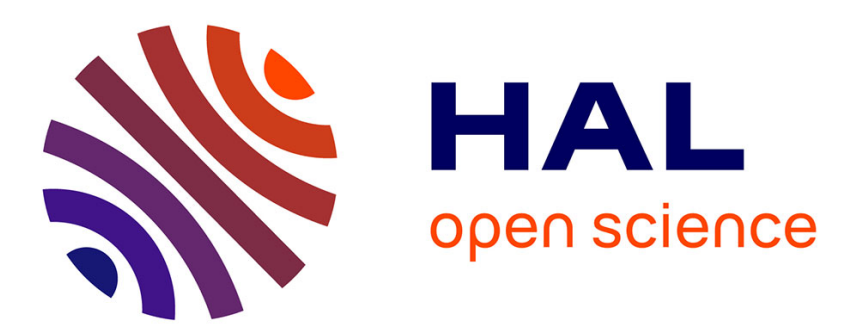

\title{
Prognostic value of tumor deposits in rectal cancer: A monocentric series of 505 patients
}

\author{
Olivier Benoit, Magali Svrcek, Ben Creavin, Morgane Bouquot, Alexandre \\ Challine, Najim Chafai, Clotilde Debove, Thibault Voron, Yann Parc, Jeremie \\ Lefevre
}

\section{To cite this version:}

Olivier Benoit, Magali Svrcek, Ben Creavin, Morgane Bouquot, Alexandre Challine, et al.. Prognostic value of tumor deposits in rectal cancer: A monocentric series of 505 patients. Journal of Surgical Oncology, In press, 10.1002/jso.26165 . hal-02961795

\section{HAL Id: hal-02961795 \\ https://hal.sorbonne-universite.fr/hal-02961795}

Submitted on 8 Oct 2020

HAL is a multi-disciplinary open access archive for the deposit and dissemination of scientific research documents, whether they are published or not. The documents may come from teaching and research institutions in France or abroad, or from public or private research centers.
L'archive ouverte pluridisciplinaire HAL, est destinée au dépôt et à la diffusion de documents scientifiques de niveau recherche, publiés ou non, émanant des établissements d'enseignement et de recherche français ou étrangers, des laboratoires publics ou privés. 
Journal of Surgical Oncology

\section{Prognostic value of tumor deposits in rectal cancer: A monocentric series of $\mathbf{5 0 5}$ patients.}

\begin{tabular}{|r|l|}
\hline Journal: & Journal of Surgical Oncology \\
\hline Manuscript ID & Draft \\
\hline Wiley - Manuscript type: & Research Article \\
\hline Author: & n/a \\
\hline Complete List of Authors: & $\begin{array}{l}\text { Benoit, Olivier; Sorbonne Universite, Assistance Publique Hôpitaux de } \\
\text { Paris, Saint Antoine University Hospital, Department of Digestive Surgery } \\
\text { Svrcek, Magali; Sorbonne Universite, Pathology } \\
\text { Creavin, Ben; Ireland East Hospital Group, Elm park } \\
\text { Bouquot, Morgane; Sorbonne Universite, Assistance Publique Hôpitaux } \\
\text { de Paris, Saint Antoine University Hospital, Department of Digestive } \\
\text { Surgery } \\
\text { Challine, Alexandre; Sorbonne Universite, Assistance Publique Hôpitaux } \\
\text { de Paris, Saint Antoine University Hospital, Department of Digestive } \\
\text { Surgery } \\
\text { Chafai, Najim; Sorbonne Universite, Assistance Publique Hôpitaux de } \\
\text { Paris, Saint Antoine University Hospital, Department of Digestive Surgery } \\
\text { Debove, Clotilde; Sorbonne Universite, Assistance Publique Hôpitaux de } \\
\text { Paris, Saint Antoine University Hospital, Department of Digestive Surgery } \\
\text { Voron, Thibault; Sorbonne Université, Assistance Publique Hôpitaux de } \\
\text { Paris, Saint Antoine University Hospital, Department of Digestive Surgery } \\
\text { parc, yann; Sorbonne Universite, Assistance Publique Hôpitaux de Paris, } \\
\text { Saint Antoine University Hospital, Department of Digestive Surgery } \\
\text { lefevre, jeremie; Sorbonne Universite, Assistance Publique Hôpitaux de } \\
\text { Paris, Saint Antoine University Hospital, Department of Digestive Surgery }\end{array}$ \\
\hline Key Words: & \begin{tabular}{l} 
rectal cancer, tumor deposits, N1c, nodal staging \\
\hline
\end{tabular} \\
\hline
\end{tabular}

\section{SCHOLARONE Manuscripts}


Prognostic value of tumor deposits in rectal cancer: A monocentric series of 505 patients.

Olivier Benoit (MD) ${ }^{1}$; Magali Svrcek (MD, PhD) ${ }^{2}$; Ben Creavin (MD) ${ }^{3}$; Morgane Bouquot (MD) ${ }^{1}$; Alexandre Challine (MD) ${ }^{1}$; Najim Chafai (MD) ${ }^{1}$; Clotilde Debove (MD) ${ }^{1}$; Thibault Voron $(\mathrm{MD}, \mathrm{PhD}){ }^{1}$; Yann Parc $(\mathrm{MD}, \mathrm{PhD})^{1}{ }^{1}$; Jeremie H. Lefevre (MD, PhD) ${ }^{1}$.

${ }^{1}$ Sorbonne Université, Department of Digestive Surgery, AP-HP, Hôpital Saint Antoine, F75012, Paris, France.

${ }^{2}$ Sorbonne Université, Department of Pathology, AP-HP, Hôpital Saint Antoine, F-75012, Paris, France.

${ }^{3}$ Department of Surgery, St Vincent's University Hospital, Elm Park, Dublin 4, Ireland

\section{Correspondance and reprint requests:}

Pr Jérémie H. Lefèvre, Department of Digestive Surgery, Hôpital Saint-Antoine, Assistance Publique Hôpitaux de Paris, Université Pierre et Marie Curie, Paris VI, 184 rue du Faubourg Saint-Antoine, 75012, Paris, France

Tel: 00331492825 47, Fax: 0033149282548

e-mail: jeremie.lefevre@aphp.fr

ORCID: https://orcid.org/0000-0001-7601-7464

\section{Original article}

Short title: Tumor deposits in rectal cancer.

Keywords: rectal cancer; tumor deposits; N1c; nodal staging.

Word count: 2811

Conflict of interest: No financial conflicts to disclose

Ethical statement: Procedures were performed in accordance with the ethical standards of institutional and/or national research committee and with the 1964 Helsinki declaration. 
Synopsis for table of Contents: Tumor deposits (TDs) were included in TNM staging in 2010 with creation of N1c category. In this study with 505 patients operated for rectal cancer, specimens with tumor deposits had a metastatic risk comparable to a $\mathrm{pN} 2$ stage which may lead to changes in adjuvant treatment. 


\begin{abstract}
Background and Objectives: It has been suggested that tumor deposits (TD) may have a worse prognosis in rectal cancer compared to colonic cancer. The aim of this study was to assess TDs prognosis in rectal cancer.
\end{abstract}

Methods: Patients who underwent total mesorectum excision for rectal adenocarcinoma (20112016) were included. A case-matched analysis was performed to assess the accurate impact of TDs for each $\mathrm{pN}$ category after exclusion of synchronous metastasis.

Results: 505 patients were included. TDs were observed in $99(19.6 \%)$ patients, $(\mathrm{pN} 1 \mathrm{c}=37$ (7.3\%)). TDs were associated with pT3-T4 stage $(\mathrm{p}=0.037)$, synchronous metastasis $(p=0.003)$, LN invasion $(p=0.041)$, VI $(p=0.001)$ and PNI $(p<0.001)$. TD was associated with a worse 3-year DFS among pN0 (51.2\% vs 79.8\%; $<<0.001)$; pN1 patients (35.2\% vs 70.1\%; $\mathrm{p}=0.004)$ but not among $\mathrm{pN} 2$ patients $(37.5 \%$ vs. $44.7 \% ; \mathrm{p}=0.499)$. After matching, $\mathrm{pN} 1 \mathrm{c}$ patients had a worse 3-year DFS compared with pN0 patients $(58.6 \%$ vs $82.4 \%$; $=0.035)$ and a tendency towards a worse DFS among N1 patients $(40.1 \%$ vs $64.2 \% ; p=0.153)$. DFS was worse when one TD was compared to one invaded LN (40.8\% vs $81.3 \%$; $<<0.001)$.

Conclusion: In rectal cancer, TDs have a metastatic risk comparable to a pN2 stage which may lead to changes in adjuvant treatment. 


\section{INTRODUCTION}

Rectal cancer accounts for around $30 \%$ of all colorectal cancer (CRC) with a significant improvement in prognosis and management seen when neoadjuvant chemoradiotherapy is used. [1-3] Adjuvant treatment is guided by pathological prognostic factors including lymph node metastasis (LNM), vascular invasion (VI), perineural invasion (PNI) and tumor deposits (TD). $[4]$

Tumor deposits were first described in 1935 as nodules of tumor cells in pericolic or perirectal fat tissue without lymph node architecture. [5] They were first included in AJCC/TNM $5^{\text {th }}$ classification in 1997 and considered as lymph nodes (LN) if their size was $>$ $3 \mathrm{~mm}$ in diameter or as a tumor extension when they measured $<3 \mathrm{~mm}$. Then the $6^{\text {th }}$ edition of TNM staging differentiated round-shaped tumor deposits as lymph nodes and spiculated ones as a tumor extension. Finally the $7^{\text {th }}$ edition in 2010 created a new $\mathrm{pN}$ category (i.e. pN1c) for adenocarcinoma with TD without concomitant LNM (N0). [6]

N1c category represent 5 to $10 \%$ of rectal cancers [4,7] with TDs seen in around $20 \%$ of all adenocarcinomas. [8] TDs are associated with poor prognostic markers such as a high T stage (T3-T4 stage) [7,9] and a higher N stage. [10,11] $60 \%$ of patients with stage IV disease have TDs [12] with a further $72 \%$ of colorectal cancer with PNI having TDs also. [7] Nagtegaal et al. previously reported in a meta-analysis that N1c tumors are associated with a worse disease-free survival and overall survival. [8] However, most of these studies were retrospective, based on pathological specimens from pre 2010 prior to the reclassification of the TNM stage. [8] It has been suggested that TDs may have worse prognosis in rectal cancer compared to colon cancer [9], although there a limited number of studies purely examining rectal cancer alone. Moreover, TDs identification after radiotherapy remains controversial. Although there are some arguments for lower survival due to TDs after neoadjuvant radiotherapy, [13] small number of studies have reported a poor prognosis in TD positive 
tumors after neoadjuvant radiotherapy [7] while others showed no difference. [14] The aim of this study was to assess TD and N1c stage tumor prognosis in rectal cancer.

\section{MATERIAL AND METHODS}

\section{Study population}

All consecutive patients who underwent total mesorectum excision (TME) for rectal adenocarcinoma in our Department between 2011 and 2016 were included from a prospective database. In order to select only true rectal cancers and avoid confusion with rectosigmoid cancers, all colorectal anastomosis not diverted by ileostomy were excluded. The primary endpoint of this study was to compare prognosis in tumor deposit positive tumors and N1c patients using the new TNM staging. The secondary endpoint was to assess TDs impact in a case-control setting.

\section{Data collection}

Clinical information concerning tumor, neoadjuvant treatment and surgical procedure was retrieved from patient charts. Histological data concerning TNM stage, TDs, vascular invasion, perineural invasion, tumor grade or differentiation were included from standardized pathological reports according to the $7^{\text {th }}$ edition of AJCC classification. [6] TDs were defined as tumor nodules in the fatty tissue of the mesorectum without lymph node (LN) structure. Local recurrence was defined as pelvic or anastomotic recurrence diagnosed by imaging or endoscopy. In cases with synchronous metastasis, distant recurrence was considered when new lesions appeared, or progression of existing disease occurred. Global recurrence was defined by the occurrence of local and/or metastatic recurrence.

\section{Neoadjuvant therapy and surgical procedure}


According to French and ESMO guidelines [1,3] preoperative radiotherapy was always discussed in multidisciplinary meetings for patients with mid and low rectal cancer. Patients with a cT3, cT4 or cN+ tumor were eligible for neoadjuvant radiotherapy. Patient with locally advanced cancer received either a 50.4 Gy radiotherapy associated with oral Capecitabine (CAP-50 protocol) or a short course protocol with 25 Gy radiotherapy. CAP-50 was preferred when a downsizing of tumor was expected to enhance circumferential resection margin. Surgery was performed 6-8 weeks after CAP-50 protocol or 1 week after short course protocol. A total mesorectum excision (TME) was always performed as previously described. [15] Most patients had a primary anastomosis, with intersphincteric resection if needed. In cases of anal sphincter invasion, an abdominoperineal excision was performed. All patients had a low anastomosis and so a diverting stoma to protect the anastomosis. Patients with stage III tumor on pathological exam received adjuvant chemotherapy based on multidisciplinary decision. Patients with N1c stage were considered as stage III.

\section{Outcomes}

Data concerning local recurrence, distant metastasis or death were collected from patient charts. Disease-free survival was defined as the time without local and/or metastatic recurrence and analyzed using the date of local and/or distant metastasis. When patients had initial metastatic disease, recurrence was considered when disease progression was observed.

\section{Case-control study}

In the case-control study, patients with synchronous metastatic disease were excluded. In each $\mathrm{pN}$ category, patients were matched according to sex, BMI, neoadjuvant radiotherapy, surgical procedure, pT stage, VI and PNI presence. A 2 for 1 pairing among pN0 patients and a 1 for 1 pairing in $\mathrm{pN} 1$ and $\mathrm{pN} 2$ patients was performed.

\section{Statistical analysis}


Statistical analysis was performed using SPSS Statistic 20 software (IBM, Armonk, NY; USA). Continuous variables were expressed as mean $+/$ - standard deviation and compared using Mann-Whitney U test. Nominal variables were expressed with percentage and compared using Chi-square test or Fisher test. Multivariate analysis included variables with statistical difference in univariate analysis with $\mathrm{p}$ value $<0.1$. Survival analysis was performed with Kaplan-Meyer curves and compared with log-rank test.

\section{RESULTS \\ Population}

A total of 505 patients were included. Clinical data of the cohort is summarized in table 1. There were $315(62.4 \%)$ men with a mean age of $63.3 \pm 12.7$ years. Neoadjuvant radiotherapy was performed in $275(54.5 \%)$ patients with 42 patients receiving a short course protocol. Abdominoperineal excision was performed in 86 (17\%) patients. Pathological examination showed a majority of pT3 $(\mathrm{N}=253 ; 50.1 \%)$ and $\mathrm{pN} 0(\mathrm{~N}=272 ; 53.9 \%)$ tumors. TDs were observed in $99(19.6 \%)$ patients, with 37 (7.3\%) patients classified as pN1c. Median follow-up was $32 \pm 22$ months with a total of $49(9.7 \%)$ deaths occurring during that period. Local recurrence was observed in 35 (6.9\%) patients. Among patients without synchronous metastasis $(n=455), 103(22.6 \%)$ had metastatic progression. In patients with synchronous metastasis $(\mathrm{n}=50), 32(64 \%)$ had metastatic progression. Among patients with synchronous metastasis, 5 $(10 \%)$ had hepatic associated surgery and $1(0.5 \%)$ had latero-aortic lymph node clearance.

Patients characteristics according to the presence of TDs

Comparisons between specimen with and without TDs are detailed in table 1. TD positive specimens were more frequently associated with pT3 and pT4 tumors $(\mathrm{p}<0.001), \operatorname{LN}(\mathrm{p}<0.001)$, 
VI $(p<0.001)$ and PNI $(p<0.001)$ (table 1). In multivariate analysis, TDs were associated with pT3-T4 tumors $(p=0.037)$, synchronous metastasis $(p=0.003)$, LNM $(p=0.041)$, VI $(p=0.025)$ and PNI $(p<0.001)$. We didn't include neoadjuvant radiotherapy in the variable as it is correlated with the $\mathrm{T}$ and $\mathrm{N}$ stages. Multivariate analysis did not show any statistical association with R1 resections. After exclusion of synchronous metastasis, TD positive tumors were also associated with pT3 and pT4 tumors $(\mathrm{p}<0.001)$, LN invasion $(\mathrm{p}<0.001)$, VI and PNI $(\mathrm{p}<0.001)$.

\section{Relationship between TDs and nodal stage}

pN1c tumors were seen in $37(7.3 \%)$ patients and were associated with pT3 and pT4 tumors $(p<0,001)$ and synchronous metastasis $(p<0.001)$. There was significantly more VI $(p=0.003)$ and PNI $(\mathrm{p}<0.001)$ in $\mathrm{pN} 1 \mathrm{c}$ patients. Among pN1 patients, the presence of TDs was associated with pT3 tumors $(p=0.011)$, VI $(p=0.004)$ and PNI $(p=0.001)$. Within $p N 2$ tumors, there was no difference in $\mathrm{pT}$ stage between TD positive and TD negative specimens. There was significantly more PNI involvement but no difference in VI (table 2).

\section{Oncological outcomes}

In the overall population, three-year overall survival (OS) was $90.5 \%$ and disease-free survival (DFS) was $66.4 \%$ (Table 3). pN1c patients had a worse DFS compared to $\mathrm{pN} 0$ patients $(51.2 \%$ vs $79.8 \% ; \mathrm{p}<0.001$ ), although there was no significant difference in in 3-year OS (figure 1). pN0 patients were associated with a worse DFS when synchronous metastasis $(n=283)$ was excluded (58.5\% vs $82.3 \%$; $=0.001)$. Among $\mathrm{pN} 1$ patients, there was no difference in OS, however, a worse DFS due to TDs was noted (35.2\% vs 70.1\%; $\mathrm{p}=0.004)$. A significantly worse 3-year DFS was seen in TD positive $\mathrm{pN} 1$ patients $(40.1 \%$ vs $74.6 \%$; $=0.007)$ when synchronous metastasis was excluded $(n=101)$, however, this was not seen in the pN2 patients (37.5\% vs $44.7 \% ; \mathrm{p}=0.499)$. There was no significant difference in 3-year OS (figure 2). 
Following exclusion of synchronous metastasis $(n=71)$, DFS was comparable $(41.1 \%$ vs $51.4 \%$; $\mathrm{p}=0.65$ ). There was no difference in adjuvant therapy among $\mathrm{pN} 1$ and $\mathrm{pN} 2$ patients due to TDs presence (table 2)

\section{Impact of TD numbers}

Patients with $\geq 4$ TDs $(\mathrm{N}=14$ ) had a worse 3-year DFS, however, this was not statistically significant. (20.6\% vs. $44.1 \%$; $\mathrm{p}=0.098)$. Furthermore, a worse 3 -year OS was seen in patient with $\geq 4$ TDs compared with those with $1-3$ TDs $(62.2 \%$ vs $82.2 \%$; $=0.087)$. Again, this was not statistically significant. Specimens with one TD versus one positive LN with TDs were examined. A worse DFS was noted in the TD group (40.8\% vs $81.3 \%$; $\mathrm{p}<0.001)$.

\section{Case-control study}

After matching, 22 TD-positive specimens were compared with 44 TD-negative patients among the pN0 population. There was 33 and 20 TD-positive specimens in $\mathrm{pN} 1$ and $\mathrm{pN} 2$ groups respectively matched with a 1:1 ratio. Patient characteristics after matching are reported in supplementary table 1. pN1c patients had a worse 3-year DFS compared with pN0 patients (58.6\% vs $82.4 \%$; $\mathrm{p}=0.035)$, with no difference in 3-year OS seen. Worse DFS was observed in the $\mathrm{pN} 1$ group although this was not significant (respectively $40.1 \%$ vs $64.2 \%$; $=0.153$ ). There was no difference in DFS among $\mathrm{pN} 2$ patients (32.9\% vs 46.5\%; $\mathrm{p}=0.858$ ) (figure 3)

\section{Addition of TDs with LN metastasis count}

Restaging of $\mathrm{N}$ stage was seen when the sum of TDs was added to LN positive patients. 19 patients changed from N1 to N2 and 2 N1c patients changed to N2 stage disease. These 21 new N2 patients had a worse DFS that was not statistically significant to the old N2 classification ( $28.7 \%$ vs $42.7 \% ; \mathrm{p}=0.644)$ and was significantly different to the previous N1 classification $(28.7 \%$ vs $58.9 \% ; \mathrm{p}=0.003)$. 


\section{Neoadjuvant chemoradiotherapy}

Among TD positive patients, 50 received CAP-50 neoadjuvant protocol and 36 did not have radiotherapy. There was no difference in 3-year OS ( $87.1 \%$ vs $75 \%$; $=0.447)$, local recurrence $(12.7 \%$ vs $3.7 \% ; p=0.188)$ or DFS $(35.7 \%$ vs $39.9 \% ; p=0.158)$ between patient that did and did not receive neoadjuvant chemoradiotherapy.

\section{DISCUSSION}

The present study found that TDs were present in nearly $20 \%$ of the 505 specimens examined, with N1c status counting for $7.3 \%$ of the patients. TDs were statistically associated with larger tumors, pT3-pT4, LN invasion, VI, PNI and, most importantly, synchronous metastasis. TDs had an important impact on DFS among N0 and N1 patients. TD prognosis was comparable to pN2 stage and did not impact N2 DFS which is associated with a poor outcome.

The incidence of TDs in the present study was similar to previous studies $[8,9,16,17]$, however, it was higher than a recently published review on TDs and N1c [18]. The results in the present study are supported by the use of standardized pathological reports that systematically mentioned TDs and other poor prognostic factors. Moreover, the present study included only patient operated after 2010 so the use of N1c status was systematically used for specimens with TDs and no lymph node invasion. The presence of TDs is different in rectal cancers and may be more frequent compared to colon cancer (roughly $15-18 \%$ in colon cancer). $[9,10]$ However, some reports have shown the proportion to be as high as $30 \%$, with a $29 \%$ rate of TDs in right sided colon tumors [19,20], although these studies only included patients prior to 2010 and discrepancies in TDs definition may explain such a difference. The present study has shown that a number of poor prognostic factors were associated with TDs, notably vascular invasion, perineural invasion and synchronous metastasis. These poor prognostic factors have been 
demonstrated in previous studies $[8,9,16,19,21,22]$ and suggest the cancer cells ability to disseminate.

The following study has shown that TDs were associated with a worse DFS due to distant metastasis, which is comparable to a pN2 stage. Significantly worse DFS was observed in N0 and $\mathrm{N} 1$ patients but not in $\mathrm{pN} 2$ patients, probably due to the worse prognosis encountered in pN2 stage disease. A worse DFS among N0 and N1 patients was confirmed after exclusion of patients with synchronous metastasis. After matching on poor prognostic parameters, the present study still observed a significantly worse DFS (24\%) due to TDs in pN0 patients (i.e N1c). Furthermore, this study has shown a $24 \%$ decrease in DFS due to TDs specifically in N1 patients, however, due to small sample sizes after matching, a non-significant difference in $\mathrm{pN} 1$ patients was seen. These studies results differs slightly from previous studies that showed a decrease in DFS and OS among LN-negative patient following chemoradiotherapy [7,21] but not in LN-positive patients. [21] While previous data showed a decrease in OS due to TDs [8], the present study did not observe any difference in OS due to TDs. This observation might be explain by a 3 -year OS of more than $80 \%$ even in N2 patients, although, such a high OS has already been reported in previous studies. [16,19]

Jin et al. have shown an impact of TD numbers on OS with a cut-off of $\geq 4$ TDs. [20] The present study showed a trend towards a worse OS and DFS with the number of TDs, although, this was not significant. While Jin et al only studied N1c patients, the present study included both N1c and LN positive patients which may impact on survival. This may be further supported by the finding in the present study that TDs alone have a poorer prognosis than a positive LN alone, with a $40 \%$ decrease in DFS. This can be explained by the fact that the pathway involved in cellular dissemination might be different for each modality. Some authors have demonstrated that TDs are due to twist mutation while LN spread is due to snail mutations. [23] 
N1c status is a peculiar status as it represents patients only with TDs and no LN invasion. N1c patients have a worse DFS of $51.2 \%$ in this study. N1c cancers were also associated with larger tumors and poor prognostic factors as previously suggested. [9] Compared to N0 or N1 patients without TDs, DFS was worse among N1c patients and comparable to N2 disease. It has already been proven that N1c status has a poor impact on DFS and OS in colorectal cancer. [9,24]

The present study has shown that TDs did not impact prognosis following neoadjuvant chemoradiotherapy. However, in rectal cancer, it remains uncertain if N1c status is useful after radiotherapy. While some authors suggest that patients with N1c grade have a poor prognosis after radiotherapy [7,21] other studies have failed to show a prognostic difference due to confusion between TDs and residual tumor. [14] There are still a number of difficulties in evaluating TDs prognosis after neoadjuvant treatment. Furthermore, neoadjuvant chemoradiotherapy is indicated for advanced tumors or $\mathrm{N}$ positive tumors, that have a high risk for TD presence but TDs are not easily diagnosed on MRI with great difficulties to assess their presence preoperatively.

Distinction between TD and invaded LN remains a challenge and is still debated among pathologists. [25] TDs may be confused for a completely replaced LN, venous invasion or even tumor spread. [18] The present study suggests that TDs must be mentioned in pathological reports and confusion between TDs and $\mathrm{LN}$ may lead to a lack of metastatic risk evaluation. Current TNM staging is suboptimal in its definition of TDs. A lot of pathologists do not support practices such as excluding nodules with evidence of underlying EMVI, LI or PNI from the pN1c category and thus downstaging a patient from stage III to stage II if reclassifying them according to the TNM 8 rather than the TNM 7 edition. Currently, French and European guidelines recommended post-operative chemotherapy for patients with stage III and IV rectal cancer. [1,3] However, these recommendations are based on expert consensus and protocols used in colon cancer. Recently, it has been suggested that in colon cancer, a longer 
duration of adjuvant chemotherapy may improve prognosis for high risk cancer. [26] With this in mind, the authors suggest that the presence of TDs should be considered when implementing adjuvant treatment regimens and protocols. Chavali et al. suggest that adjuvant radiotherapy improves prognosis for patients with TDs who did not receive neoadjuvant radiotherapy. [27]

The results in the present study are strengthened by a large sample size and standardized pathological reports. Furthermore, patients were only included after 2010 which corresponded to the introduction of the $7^{\text {th }}$ edition of TNM staging. However, there are still several limitations. It is a retrospective study with the majority of patients having N0 disease. TDs were present in almost $20 \%$ of the population, however, only 37 patients were classified as N1c which lead to a lack of statistical strength for this sub-group. Due to the small numbers of N1 patients after matching, a significant impact on DFS due to TDs could not be ascertained. Furthermore, the majority of patients had $<4$ TDs, again limiting the true impact of TD numbers on DFS outcomes.

\section{CONCLUSION}

In rectal cancer, TDs are a poor prognostic factor with a higher risk of metastatic recurrence comparable to $\mathrm{N} 2$ disease. Adjustments in chemotherapy protocols must be discussed for patients classified as N1c or N1 with TDs. Further large scale, multicentre, prospective studies are required to ascertain the prognostic implications of TDs.

\section{REFERENCES}

1. Glynne-Jones R, Wyrwicz L, Tiret E, et al.: Rectal cancer: ESMO Clinical Practice Guidelines for diagnosis, treatment and follow-up. Ann Oncol 2017;28:iv22-iv40. 
2. Peeters KC, Marijnen CA, Nagtegaal ID, et al.: The TME trial after a median followup of 6 years: increased local control but no survival benefit in irradiated patients with resectable rectal carcinoma. Ann Surg 2007;246:693-701.

3. Lakkis Z, Manceau G, Bridoux V, et al.: Management of rectal cancer: the 2016 French guidelines. Colorectal Dis 2017;19:115-122.

4. Chen VW, Hsieh MC, Charlton ME, et al.: Analysis of stage and clinical/prognostic factors for colon and rectal cancer from SEER registries: AJCC and collaborative stage data collection system. Cancer 2014;120 Suppl 23:3793-3806.

5. Gabriel WB, Dukes C, Bussey HJ: Lymphatic spread in cancer of the rectum. Br J Surg 1935.

6. Sobin LH, Gospodarowicz MK, Wittekind C, cancer IUa: "TNM classification of malignant tumours. 7th ed.": Wiley-Blackwell, 2010.

7. Wei XL, Qiu MZ, Zhou YX, et al.: The clinicopathologic relevance and prognostic value of tumor deposits and the applicability of N1c category in rectal cancer with preoperative radiotherapy. Oncotarget 2016;7:75094-75103.

8. Nagtegaal ID, Knijn N, Hugen N, et al.: Tumor Deposits in Colorectal Cancer: Improving the Value of Modern Staging-A Systematic Review and Meta-Analysis. J Clin Oncol 2017;35:1119-1127.

9. Bouquot M, Creavin B, Goasguen N, et al.: Prognostic value and characteristics of N1c colorectal cancer. Colorectal Dis 2018;20:O248-O255.

10. Goldstein NS, Turner JR: Pericolonic tumor deposits in patients with T3N+MO colon adenocarcinomas: markers of reduced disease free survival and intra-abdominal metastases and their implications for TNM classification. Cancer 2000;88:2228-2238.

11. Ueno H, Mochizuki H: Clinical significance of extrabowel skipped cancer infiltration in rectal cancer. Surg Today 1997;27:617-622. 
12. Puppa G, Maisonneuve P, Sonzogni A, et al.: Pathological assessment of pericolonic tumor deposits in advanced colonic carcinoma: relevance to prognosis and tumor staging. Mod Pathol 2007;20:843-855.

13. Lord AC, Graham Martinez C, D'Souza N, et al.: The significance of tumour deposits in rectal cancer after neoadjuvant therapy: a systematic review and meta-analysis. Eur J Cancer 2019;122:1-8.

14. Song JS, Chang HJ, Kim DY, et al.: Is the N1c category of the new American Joint Committee on cancer staging system applicable to patients with rectal cancer who receive preoperative chemoradiotherapy? Cancer 2011;117:3917-3924.

15. Bennis M, Parc Y, Lefevre JH, et al.: Morbidity risk factors after low anterior resection with total mesorectal excision and coloanal anastomosis: a retrospective series of 483 patients. Ann Surg 2012;255:504-510.

16. Gopal P, Lu P, Ayers GD, et al.: Tumor deposits in rectal adenocarcinoma after neoadjuvant chemoradiation are associated with poor prognosis. Mod Pathol 2014;27:1281-1287.

17. Tong LL, Gao P, Wang ZN, et al.: Is the seventh edition of the UICC/AJCC TNM staging system reasonable for patients with tumor deposits in colorectal cancer? Ann Surg 2012;255:208-213.

18. Jin M, Frankel WL: Lymph Node Metastasis in Colorectal Cancer. Surg Oncol Clin N Am 2018;27:401-412.

19. Shimada Y, Takii Y: Clinical impact of mesorectal extranodal cancer tissue in rectal cancer: detailed pathological assessment using whole-mount sections. Dis Colon Rectum 2010;53:771-778. 
20. Jin M, Roth R, Rock JB, et al.: The impact of tumor deposits on colonic adenocarcinoma AJCC TNM staging and outcome. Am J Surg Pathol 2015;39:109115.

21. Wang Y, Zhang J, Zhou M, et al.: Poor prognostic and staging value of tumor deposit in locally advanced rectal cancer with neoadjuvant chemoradiotherapy. Cancer Med 2019;8:1508-1520.

22. Ueno H, Mochizuki H, Hashiguchi Y, et al.: Extramural cancer deposits without nodal structure in colorectal cancer: optimal categorization for prognostic staging. Am J Clin Pathol 2007;127:287-294.

23. Fan XJ, Wan XB, Yang ZL, et al.: Snail promotes lymph node metastasis and Twist enhances tumor deposit formation through epithelial-mesenchymal transition in colorectal cancer. Hum Pathol 2013;44:173-180.

24. Yamano T, Semba S, Noda M, et al.: Prognostic significance of classified extramural tumor deposits and extracapsular lymph node invasion in T3-4 colorectal cancer: a retrospective single-center study. BMC Cancer 2015;15:859.

25. Rock JB, Washington MK, Adsay NV, et al.: Debating deposits: an interobserver variability study of lymph nodes and pericolonic tumor deposits in colonic adenocarcinoma. Arch Pathol Lab Med 2014;138:636-642.

26. Lieu C, Kennedy EB, Bergsland E, et al.: Duration of Oxaliplatin-Containing Adjuvant Therapy for Stage III Colon Cancer: ASCO Clinical Practice Guideline. J Clin Oncol 2019;37:1436-1447.

27. Chavali LB, Llanos AAM, Yun JP, et al.: Radiotherapy for Patients With Resected Tumor Deposit-Positive Colorectal Cancer: A Surveillance, Epidemiology, and End Results-Based Population Study. Arch Pathol Lab Med 2018;142:721-729. 


\section{Availability of data}

The data that support the findings of this study are available on request from the corresponding author. The data are not publicly available due to privacy or ethical restrictions.

\section{FIGURES LEGEND}

Figure 1: Survival curves between pNO and pN1c patients. A: OS; B: DFS

Figure 2: Survival curves within LN invasion positive specimen. A: N1; B: N2. TD neg: No TDs; TD pos: TD presence

Figure 3: Survival curves after matching. A: pN0; B: pN1; C: pN2. TD neg: No TDs; TD pos: TDs presence 


\begin{tabular}{|c|c|c|c|c|c|c|c|c|}
\hline & & \multirow[b]{2}{*}{ Total $(\mathrm{N}=505)$} & \multirow[b]{2}{*}{ TD pos $(N=99)$} & \multirow[b]{2}{*}{ TD neg(N=406) } & \multirow[b]{2}{*}{ p } & \multicolumn{3}{|c|}{ Multivariate } \\
\hline & & & & & & OR & IC[95\%] & $\mathbf{p}$ \\
\hline Age (years) & & $63.3( \pm 12.7)$ & $63.8( \pm 12.5)$ & $63.2( \pm 12.8)$ & 0.647 & NI & & \\
\hline Sex & M & $315(62.4)$ & $56(56.6)$ & $259(63.8)$ & 0.203 & NI & & \\
\hline BMI (kg/m2) & & $25.4(+/-4.8)$ & $25.2( \pm 4.6)$ & $25.5( \pm 4.9)$ & 0.634 & NI & & \\
\hline Synchronous M+ & & $48(9.5)$ & & & & NI & & \\
\hline Neoadjuvant & RCT & $233(46.1)$ & $50(50.5)$ & $183(45.1)$ & 0.099 & NI & & \\
\hline \multirow[t]{3}{*}{ TTT } & RT & $42(8.3)$ & $13(13.1)$ & $29(7.1)$ & & & & \\
\hline & Chemotherapy & $12(2.4)$ & $3(3)$ & $9(2.2)$ & & & & \\
\hline & TEM & $16(3.2)$ & $1(1)$ & $15(3.7)$ & & & & \\
\hline \multirow[t]{5}{*}{ Surgery } & CRA & $38(7.5)$ & $8(8.1)$ & $30(7.4)$ & 0.226 & NI & & \\
\hline & CAA & $358(70.9)$ & $65(65.7)$ & $293(72.2)$ & & & & \\
\hline & APE & $86(17)$ & $23(23.2)$ & $63(15.5)$ & & & & \\
\hline & IPAA & $18(3.6)$ & $1(1)$ & $17(4.2)$ & & & & \\
\hline & Hartman & $5(1)$ & $2(2)$ & $3(0.7)$ & & & & \\
\hline \multirow[t]{5}{*}{ pT } & $\mathbf{0}$ & $11(2.2)$ & 0 & $11(2.7)$ & $<0.001 *$ & 2.24 & $1.05-4.79$ & 0.037 \\
\hline & 1 & $43(8.5)$ & $2(2)$ & $41(10.1)$ & & & & \\
\hline & 2 & $140(27.7)$ & $11(11)$ & $129(31.8)$ & & & & \\
\hline & 3 & $253(50.1)$ & $69(69.7)$ & $184(45.3)$ & & & & \\
\hline & 4 & $58(11.5)$ & $17(17.2)$ & $41(10.1)$ & & & & \\
\hline \multirow[t]{3}{*}{ pN } & $\mathbf{0}$ & $272(53.9)$ & $37(37.4)$ & $272(67)$ & $<0.001 * *$ & 1.83 & $1.02-3.28$ & 0.041 \\
\hline & 1 & $110(21.8)$ & $37(37.4)$ & $73(18)$ & & & & \\
\hline & 2 & $86(17)$ & $25(25.3)$ & $61(15)$ & & & & \\
\hline M1 pathological & & $50(9.9)$ & $23(23.2)$ & $27(6.7)$ & $<0.001$ & 3.17 & $1.49-6.78$ & 0.003 \\
\hline \multirow{2}{*}{ Resection status } & R1 & $69(13.7)$ & $28(28.3)$ & $41(10.1)$ & $<0.001$ & NS & & \\
\hline & R2 & $1(0.2)$ & 0 & $1(0.2)$ & & & & \\
\hline VI & & $234(46.3)$ & $72(72.7)$ & $162(39.9)$ & $<0.001$ & 2.07 & $1.10-3.90$ & 0.025 \\
\hline PNI & & $152(30.1)$ & $64(64.6)$ & 88 (21.7) & $<0.001$ & 2.70 & $1.48-4.92$ & $<0.001$ \\
\hline \multirow[t]{2}{*}{ Grade } & Low & $394(78)$ & $70(84.3)$ & $324(96.1)$ & $<0.001$ & 2.99 & $1.16-7.72$ & 0.023 \\
\hline & High & $26(5.1)$ & $13(15.7)$ & $13(3.9)$ & & & & \\
\hline Adjuvant TTT & & $223(44.2)$ & $85(85.9)$ & $138(34)$ & $<\mathbf{0 . 0 0 1}$ & NI & & \\
\hline
\end{tabular}


TD: Tumor deposit; CRT: Chemoradiotherapy (CAP-50 protocol); RT: Short course radiotherapy; CRA : Colo-rectal anastomosis; CAA: Colo-anal anastomosis; IPAA: Ileal pouch anal anastomosis; APE: Abdominoperineal excision; VI: Vascular invasion; PNI: Perineural invasion; TTT : treatment. 


\begin{tabular}{|c|c|c|c|c|c|c|c|c|c|c|c|c|c|}
\hline & & & No & & & & N1 & & & & $\mathbf{N 2}$ & & \\
\hline & & $\begin{array}{c}\text { Total } \\
(\mathrm{N}=309)\end{array}$ & $\begin{array}{c}\text { N1c } \\
(\mathrm{N}=37)\end{array}$ & $\begin{array}{c}\text { TD neg } \\
(\mathrm{N}=272)\end{array}$ & $\mathbf{p}$ & $\begin{array}{c}\text { Total } \\
(\mathrm{N}=110)\end{array}$ & $\begin{array}{l}\text { TD pos } \\
(\mathrm{N}=37)\end{array}$ & $\begin{array}{l}\text { TD neg } \\
(\mathrm{N}=73)\end{array}$ & $\mathbf{p}$ & $\begin{array}{c}\text { Total } \\
(\mathrm{N}=\mathbf{8 6})\end{array}$ & $\begin{array}{l}\text { TD pos } \\
(\mathrm{N}=25)\end{array}$ & $\begin{array}{l}\text { TD neg } \\
(\mathrm{N}=61)\end{array}$ & $\mathbf{p}$ \\
\hline Neoadjuvant & $\mathbf{0}$ & $117(37.9)$ & $8(21.6)$ & $109(40.1)$ & 0.099 & $47(42.7)$ & $14(37.8)$ & $33(45.2)$ & 0.323 & $36(41.9)$ & $10(40.0)$ & $26(42.6)$ & 0.752 \\
\hline radiotherapy & CRT & $156(50.2)$ & $26(70.2)$ & $130(47.8)$ & & $44(40)$ & $16(43.2)$ & $28(38.4)$ & & $33(38.4)$ & $8(32.0)$ & $25(41.0)$ & \\
\hline & RT & $19(6.1)$ & $2(5.4)$ & $17(6.2)$ & & $14(12.7)$ & $7(18.9)$ & $7(9.6)$ & & $9(10.5)$ & $4(16.0)$ & $5(8.2)$ & \\
\hline Surgery & CRA & $19(6.1 \%)$ & $1(2.7)$ & $18(6.6)$ & 0.274 & $9(8.2 \%)$ & $3(8.1)$ & $6(8.2)$ & 0.275 & $10(11.6)$ & $4(16.0)$ & $6(9.8)$ & 0.263 \\
\hline & CAA & $221(71.5)$ & $24(64.9)$ & $197(72.4)$ & & $77(70)$ & $26(70.3)$ & $51(69.9)$ & & $60(69.8)$ & $15(60.0)$ & $45(73.8)$ & \\
\hline & APE & $59(19.1)$ & $12(32.4)$ & $47(17.3)$ & & $15(13.6)$ & $5(13.5)$ & $10(13.7)$ & & $12(14.0)$ & $5(13.5)$ & $10(13.7)$ & \\
\hline pT & $\mathbf{0}$ & $10(3.2)$ & 0 & $6(2.2)$ & $<0.001$ & $2(1.8)$ & 0 & $2(2.7)$ & 0.011 & $3(3.5)$ & 0 & $3(4.9)$ & 0.636 \\
\hline & 1 & $31(10.0)$ & 0 & $34(12.5)$ & & $5(4.5)$ & $1(2.7)$ & $4(5.5)$ & & $4(4.7)$ & $1(4.0)$ & $3(4.9)$ & \\
\hline & 2 & $101(32.7)$ & $4(10.8)$ & $98(36)$ & & $30(27.3)$ & $5(13.5)$ & $25(34.2)$ & & $8(9.3)$ & $2(8.0)$ & $6(9.8)$ & \\
\hline & 3 & $142(46)$ & $26(70.3)$ & $116(42.6)$ & & $60(54.5)$ & $29(78.4)$ & $31(42.5)$ & & $51(59.3)$ & $14(56.0)$ & $37(60.7)$ & \\
\hline & 4 & $25(8.1)$ & $7(18.9)$ & $18(6.6)$ & & $13(11.8)$ & $2(5.4)$ & $11(15.1)$ & & $20(23.3)$ & $8(32.0)$ & $12(19.7)$ & \\
\hline pM1 & & $26(8.4)$ & $14(37.8)$ & $12(4.4)$ & $<0.001$ & $9(8.2)$ & $4(10.8)$ & $5(6.8)$ & 0.481 & $15(17.4)$ & $5(20.0)$ & $10(16.4)$ & 0.689 \\
\hline Resection & R1 & $31(10.0)$ & $9(24.3)$ & $22(8.1)$ & 0.008 & $20(18.2)$ & $26(70.3)$ & $64(87.7)$ & 0.036 & $18(20.9)$ & $8(32.0)$ & $10(16.4)$ & 0.144 \\
\hline status & $\mathbf{R 2}$ & $1(0.3)$ & 0 & $1(0.4)$ & & 0 & 0 & 0 & & 0 & 0 & 0 & \\
\hline VI & & $106(34.3)$ & $21(56.8)$ & $85(31.2)$ & 0.003 & $65(59.1)$ & $29(78.4)$ & $36(49.3)$ & 0.004 & $63(73.3)$ & $22(88.0)$ & $41(67.2)$ & 0.062 \\
\hline PNI & & $53(17.2)$ & $18(48.6)$ & $35(12.9)$ & $<0.001$ & $46(41.8)$ & $24(64.9)$ & $22(30.1)$ & 0.001 & $53(61.6)$ & $22(88.0)$ & $31(50.8)$ & 0.001 \\
\hline Grade & Low & $250(80.9)$ & $28(90.3)$ & $222(97.8)$ & 0.058 & $82(74.5)$ & $26(86.7)$ & $56(91.8)$ & 0.470 & $62(72.1)$ & $16(72.7)$ & 46 (93.9) & 0.021 \\
\hline & High & $8(2.6)$ & $3(9.7)$ & $5(2.2)$ & & $9(8.2)$ & $4(13.3)$ & $5(8.2)$ & & $9(10.5)$ & $6(27.3)$ & $3(6.1)$ & \\
\hline Adjuvant TTT & & $53(17.2)$ & $30(81.1)$ & $23(8.5)$ & $<0.001$ & $95(86.4)$ & $33(88.2)$ & $62(84.9)$ & 0.77 & $75(87.2)$ & $22(88.0)$ & $53(86.9)$ & 1 \\
\hline
\end{tabular}

Table 2. Comparison between TD positives and negatives specimen according to LN involvement.

TD: Tumor deposit; CRT: Chemoradiotherapy (CAP-50); RT: Short course Radiotherap; VI: Vascular invasion; PNI: Perineural invasion; TTT : Treatment. 


\begin{tabular}{|c|c|c|c|c|c|c|c|c|}
\hline & OS (\%) & $\mathbf{p}$ & DFS (\%) & $\mathbf{p}$ & LR (\%) & p & DM (\%) & $\mathbf{p}$ \\
\hline Population & 90.5 & & 66.4 & & 92.3 & & 68.9 & \\
\hline pNo & 94.6 & & 79.8 & & 92.4 & & 83.1 & \\
\hline pN1c & 89.7 & & 51.2 & & 87.1 & & 55.1 & \\
\hline pN1 & 90 & & 58.9 & & 92.4 & & 60 & \\
\hline $\mathrm{pN} 1 / \mathrm{TD}$ pos & 80.8 & 0.389 & 35.2 & 0.004 & 93.1 & 0.737 & 35 & 0.003 \\
\hline pN1/TD neg & 93.2 & & 70.1 & & 92.1 & & 72 & \\
\hline pN2 & 80.6 & & 42.7 & & 94 & & 44.1 & \\
\hline $\mathrm{pN} 2 / \mathrm{TD}$ pos & 76.4 & 0.438 & 37.5 & 0.499 & 100 & 0.234 & 37.5 & 0.375 \\
\hline pN2/TD neg & 82.1 & & 44.7 & & 92 & & 46.6 & \\
\hline \multicolumn{9}{|l|}{ M0 } \\
\hline TD pos & 89.5 & 0.422 & 46.7 & $<0.001$ & 92.7 & 0.825 & 47.8 & $<0.001$ \\
\hline TD neg & 92.5 & & 76.5 & & 93.4 & & 76.8 & \\
\hline 1 TD & 82 & 0.053 & 40.8 & $<0.001$ & 79.1 & 0.220 & 47.6 & 0.001 \\
\hline N1a/TD ng & 95.2 & & 81.3 & & 93.4 & & 81.3 & \\
\hline \multicolumn{9}{|l|}{ TD number } \\
\hline$\geq 4$ & 62.2 & 0.087 & 20.6 & 0.098 & 83.3 & 0.444 & 20.6 & 0.96 \\
\hline$<4$ & 86 & & 44.1 & & 93.3 & & 45.3 & \\
\hline
\end{tabular}

Table 3: Three-year survival.

OS: Overall survival; DFS: Disease-free survival; LR: Local recurrence free survival; DM: Distant metastasis free survival 

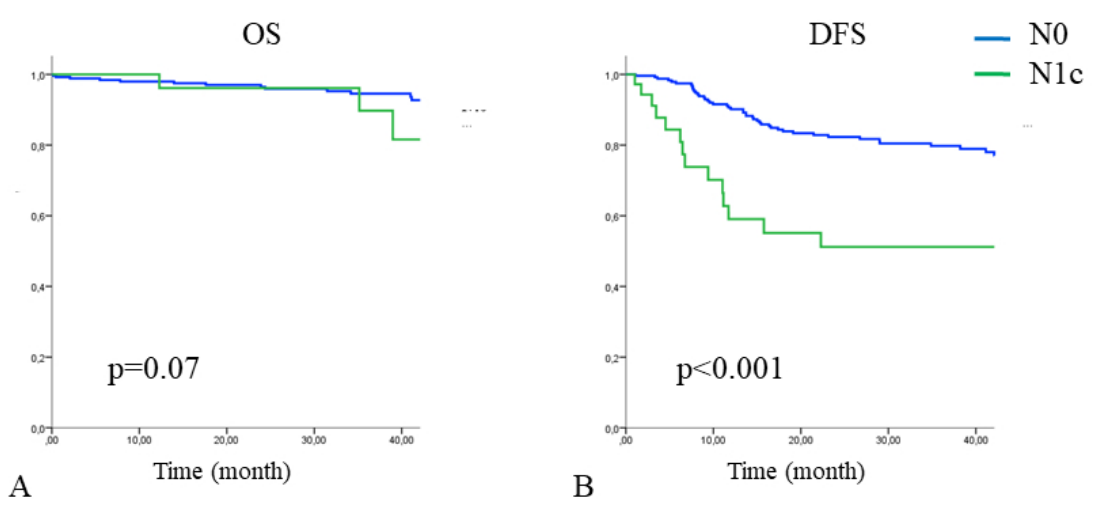

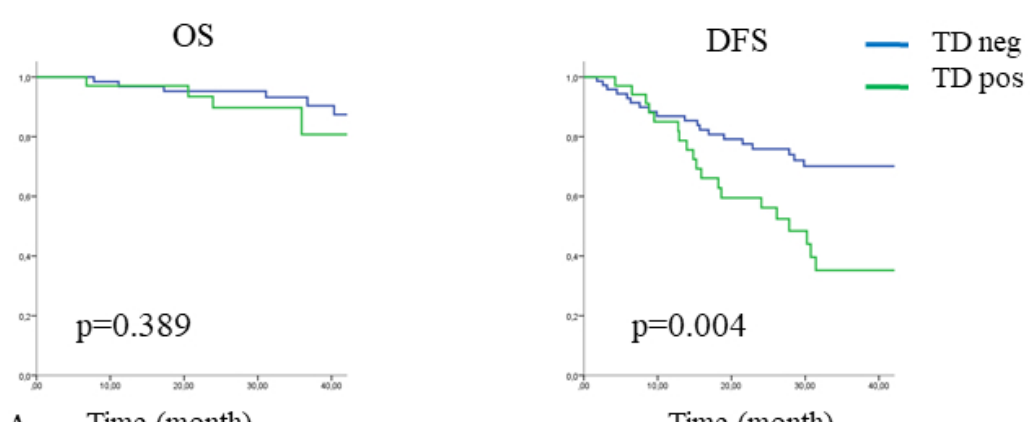

A Time (month)

Time (month)

OS

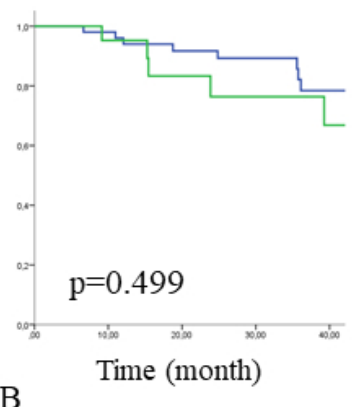

DFS

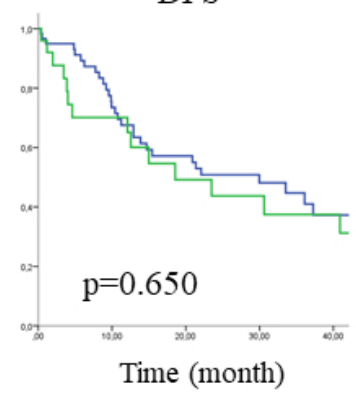

B 

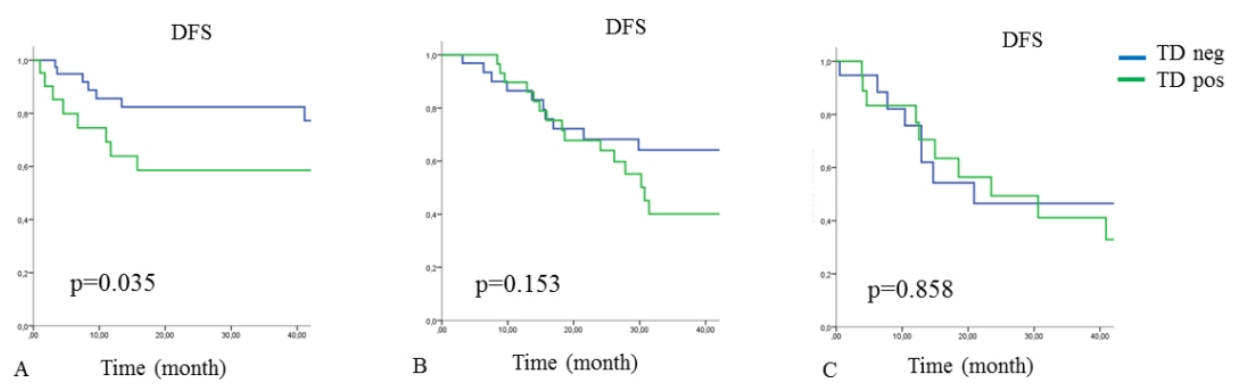

$319 \times 203 \mathrm{~mm}(96 \times 96$ DPI $)$

John Wiley \& Sons, Inc. 


\begin{tabular}{|c|c|c|c|c|c|c|c|c|c|c|}
\hline & & & No & & & N1 & & & N2 & \\
\hline & & $\begin{array}{l}\text { TD pos } \\
(\mathrm{N}=22)\end{array}$ & $\begin{array}{l}\text { TD neg } \\
(\mathrm{N}=44)\end{array}$ & $\mathbf{p}$ & $\begin{array}{l}\text { TD pos } \\
(\mathrm{N}=33)\end{array}$ & $\begin{array}{l}\text { TD neg } \\
(\mathrm{N}=33)\end{array}$ & $\mathbf{p}$ & $\begin{array}{l}\text { TD pos } \\
(\mathrm{N}=\mathbf{2 0})\end{array}$ & $\begin{array}{l}\text { TD neg } \\
(\mathrm{N}=\mathbf{2 0})\end{array}$ & $\mathbf{p}$ \\
\hline Age* (years) $^{*}$ & & $64.9( \pm 10.5)$ & $65.3( \pm 14.3)$ & 0.914 & $63.6( \pm 14.4)$ & $59.9( \pm 14.0)$ & 0.296 & $65.7( \pm 10.7)$ & $60.2( \pm 11.4)$ & 0.128 \\
\hline Sex* & $\mathbf{M}$ & $11(50)$ & $22(50)$ & 1 & $17(51.5)$ & $17(51.7)$ & 1 & $13(65)$ & $13(65)$ & 1 \\
\hline BMI* $\left(\mathrm{kg} / \mathrm{m}^{2}\right)$ & & $25.3( \pm 4.5)$ & $25.7( \pm 4.7)$ & 0.764 & $24.9( \pm 5.3)$ & $24.3( \pm 5.8)$ & 0.722 & $26.6( \pm 4.1)$ & $26.5( \pm 6.3)$ & 0.961 \\
\hline Neoadjuvant & $\mathbf{0}$ & $8(36.4)$ & $16(36.4)$ & 0.331 & $11(33.3)$ & $13(39.4)$ & 0.601 & $10(50)$ & $10(50)$ & 0,896 \\
\hline radiotherapy * & CRT & $14(63.6)$ & $24(54.5)$ & & $15(45.5)$ & $16(48.5)$ & & $7(35)$ & $6(30)$ & \\
\hline & RT & 0 & $4(9.1)$ & & $7(21.2)$ & $4(12.1)$ & & $3(15)$ & $4(20)$ & \\
\hline Surgery* & CRA & $1(4.5)$ & $3(6.8)$ & 0.905 & $2(6.1)$ & $2(6.1)$ & 0.907 & $2(10)$ & $2(10)$ & 1 \\
\hline & CAA & $16(72.7)$ & $30(68.2)$ & & $24(72.7)$ & $25(75.8)$ & & $13(65)$ & $13(65)$ & \\
\hline & APE & $5(22.7)$ & $11(25)$ & & $5(15.2)$ & $5(15.2)$ & & $5(25)$ & $5(25)$ & \\
\hline pT * & 1 & 0 & 0 & 1 & $1(3)$ & $1(3)$ & 1 & $1(5)$ & $1(5)$ & 1 \\
\hline & 2 & $4(18.2)$ & $8(18.2)$ & & $5(15.2)$ & $5(15.2)$ & & $2(10)$ & $2(10)$ & \\
\hline & 3 & $13(59.1)$ & $26(59.1)$ & & $25(75.8)$ & $25(75.8)$ & & $11(55)$ & $11(55)$ & \\
\hline & 4 & $5(22.7)$ & $10(22.7)$ & & $2(6.1)$ & $2(6.1)$ & & $6(30)$ & $6(30)$ & \\
\hline Resection status & $\mathbf{R 1}$ & $6(27.3)$ & $8(18.2)$ & 0.524 & $11(33.3)$ & $5(15.2)$ & 0.150 & $7(35)$ & $5(25)$ & 0,731 \\
\hline VI* & & $12(54.5)$ & $23(52.3)$ & 1 & $25(75.8)$ & $21(63.6)$ & 0.422 & $17(85)$ & $17(85)$ & 1 \\
\hline PNI* & & $12(54.5)$ & $16(36.4)$ & 0.192 & $20(60.6)$ & $13(39.4)$ & 0.139 & $17(85)$ & $12(60)$ & 0.155 \\
\hline Adjuvant TTT & & $16(72.7)$ & $4(9.1)$ & $<0.001$ & $29(87.9)$ & $28(84.8)$ & 1 & $17(85)$ & $17(85)$ & 1 \\
\hline
\end{tabular}

Supplementary table 1. Patients characteristics after matching.

*: pairing factors.

TD: Tumor deposit; CRT: Chemoradiotherapy (CAP-50); RT: Short course Radiotherap; VI: Vascular invasion; PNI: Perineural invasion;TTT : Treatment. 